\title{
Wiwaldis Wort zu Wortspielen
}

Dies ist das perfekte Buch für mich! Schließlich leide ich unter Spielsucht... Wortspielsucht.

Das scheint bei uns Hunden im Blut zu liegen. Mein Onkel war ebenfalls spielsüchtig. Er war ein Zocker-Spaniel! Oje... ich merke, es geht schon wieder los! Die meisten meiner Wortspiele entstehen übrigens nicht durch Wörter, sondern durch Zeichnungen... hier die schlimmsten Wortspiel-Kritzeleien aus meinem Skizzenbuch...

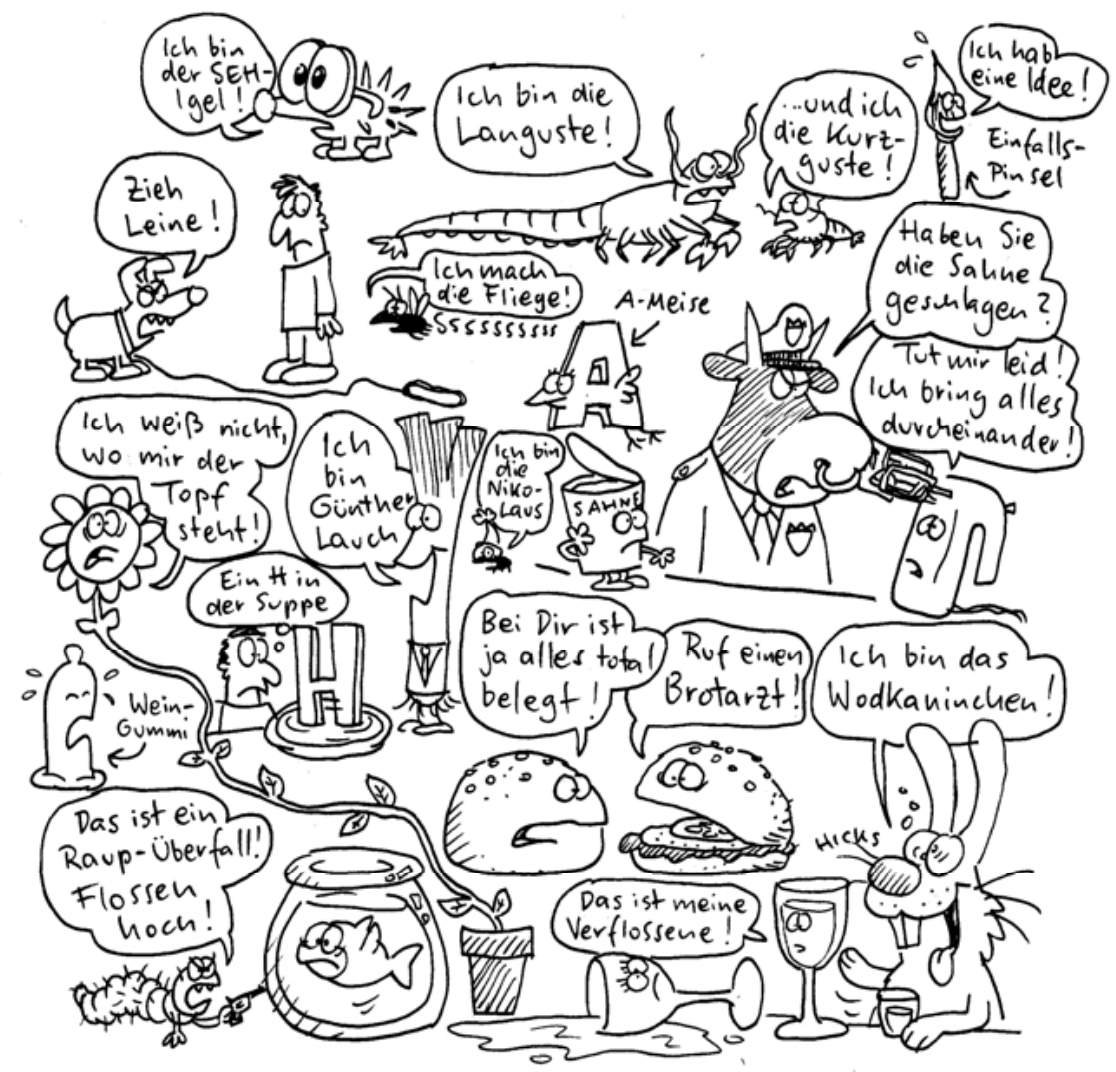

Wortspielhölle (@ Martin Reinl) 


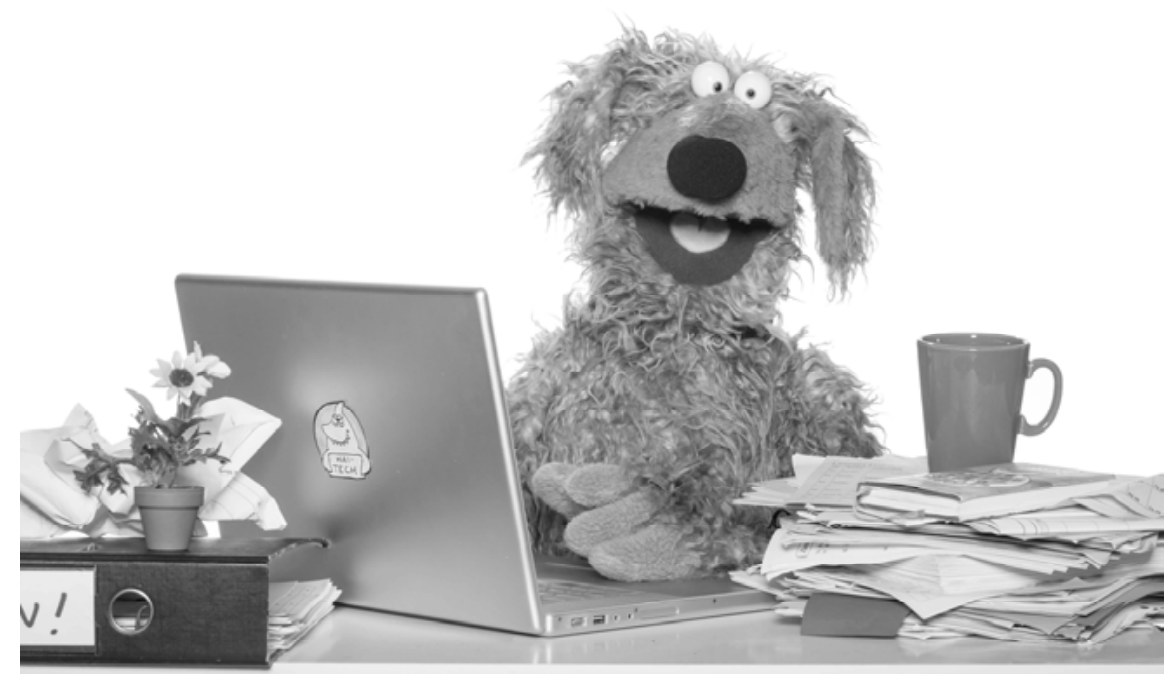

Wiwaldi (@ bigSmile) 



\section{Personenindex}

Aischylos 77

Alan, Ray 98

Aristoteles 145, 174

Arp, Hans 187, 294, 298

Astor, Willy 7, 12, 107, 117, 205, 208, 213f., 216, 226, 250, 299, 306

Bachtin, Michail 125

Bailly, Sébastien $250 f$.

Ball, Hugo 57, 61ff.

Benyoëtz, Elazar 12, 143ff., 148ff., 285

Bernstein, F. W. 72, 200

Böttcher, Bas 5, 9, 45ff., 234, 292ff., 296f., 299, 314

Breton, André 18

Bruegel, Pieter 18

Brunke, Timo 234

Busch, Wilhelm 189

Claudel, Paul 311

Clausen, Murmel 5, 9f., 223ff., 248, 250, 293

Conti, Nina 101

Dostojewski, Fjodor 22

Dunham, Jeff 96, 98

Eco, Umberto 256, 302

Eilert, Bernd 225

Erhardt, Heinz 7, 70, 106f., 117, 140f., 158f., 175, 198, 234, 244, 274, 294, 301, 307f.

Fesl, Fredl 225

Feuerstein, Herbert 274, 281, 274, 281

Fontane, Theodor 73

Franc-Nohain 251

Freud, Sigmund 22, 249

George, Stefan 143

Gernhardt, Robert 8f., 48, 67, 69f., 74, 119, 141, 158f., 225, 234, 244, 302

Gide, André 129

Goethe, Johann Wolfgang von $18,147,149$ f. Grammel, Sascha 95f., 98, $100 \mathrm{ff}$.

Grass, Günter 21
Grimm, Jacob und Wilhelm 5, 77, 86, 316

Harsdörffer, Georg Philipp 293

Hartling, Pit 116

Hausmann, Raoul 60

Haydn, Joseph 147

Hegel, Georg Wilhelm Friedrich 174

Heidegger, Martin 143, 311

Herbig, Michael „Bully“ 223, 227

Hieronymus 149

Hippocrates 95

Hirdes, Christian 5ff., 9, 12, 105ff., 121, 123, 127f., 133ff., 139ff., 247, 285, 293, 299f., $316 \mathrm{ff}$.

Hoffmann, E. T. A. 22

Hogekamp, Wolf 45

Hohler, Franz 5, 7, 9, 174, 185ff., 248, 285 , 301, 315

Huelsenbeck, Richard 60f., 63

Humboldt, Wilhelm von 149

Hüsch, Hanns Dieter 77

Insterburg \& Co. 169

Jandl, Ernst 21f., 46, 48, 68, 186, 190, 193

Jará, Jörg 95, 98, 100ff.

Jelinek, Elfriede 28

Joyce, James 69

Kant, Immanuel $173 \mathrm{f}$.

Karlstadt, Liesl 191

Kästner, Erich 244

Kaut, Ellis 286, 293

Keller, Gottfried 149

King, Kolby $97 f$.

Kishon, Ephraim 141

Kraus, Karl 71, 311

Kreisler, Georg 117

Krüger, Mike 107, 169

Kulička, Bronko 225

Luther, Martin 144, 149, 154 
Malmsheimer, Jochen 5, 11, 75ff., 86, 88ff., 92

Mann, Thomas $19 f$.

Marley, Bob 142

Marx, Karl 18

Moreno, Peter 98

Morgenstern, Christian 48, 70, 123, 159, 187, 198, 294

Moszkowski, Alexander 67

Nacken, Jakob 5, 9, $231 \mathrm{ff}$.

Nietzsche, Friedrich 174

Ono, Yoko 112

Paul, Hermann 121

Pflüger, Andreas 9, 223, 248

Pipovič, Pavel 225

Platon 95, 145, 173f., 288

Plinius der Jüngere $13,255 f f$.

Plutarch 95

Poier-Bernhard, Astrid 294

Polt, Gerhard 186

Pratchett, Terry 169

Pumuckl 235, $286 f$.

Queneau, Raymond 301f.

Reinl, Martin 5, 10, 13, 249f., 271ff., 271ff., $302 f$.

Richaud, André de 250

Richter, Adrian Ludwig 82

Ringelnatz, Joachim 46, 48, 70, 159, 187, 198, 294

Roche, Paul 266

Rückert, Friedrich 88, 92

Rühm, Gerhard 70

Rühmkorf, Peter 21

Ruquier, Laurent 250

Sachs, Hans 149

Saussure, Ferdinand de 145, 288ff., 292

Scharrenberg (Scharri), Philipp 5, 7, 9, 12, 117, 167ff., 178, 294, 298, 301
Schiller, Friedrich 132, 188

Schindler, George 97

Schobert \& Black 169

Schönen, Michael 5, 8, 12, 67, 117, 157ff., 174, 247, 249, 294

Schürmann-Mock, Iris 5, 8, 114, 195ff., 305

Schwitters, Kurt 57ff., 62, $64 \mathrm{f}$.

Seidel, Heinrich 70

Shakespeare, William 22, 31f., 42, 48

Sokrates 174, 288

Sommerstorff, Otto 68

Sophokles 22

Spencer, Bud 157

Steinhöfel, Andreas 315

Tabori, George 77

Tacitus, Publius Cornelius 256, 261

Tawada, Yoko 4, 8, 11, 13, 17ff., 32, 35, 255 , $268,306,312,316$

Thun, Helge 5, 9f., 117, 241ff., 313

Till \& Obel 107

Tomkins, Benjamin 95, $100 \mathrm{ff}$.

Tschirner, Nora 224

Tzara, Tristan 60f., 65

Valentin, Karl 191

Valéry, Paul $152 \mathrm{f}$.

Vierck, Kai 227

Waalkes, Otto 48, 107, 141, 169, 225, 244

Walter, Otto F. 193

Wartke, Bodo 5, 8f., 12, 115ff., 123, 129, 133 , 135, 174, 247, 285, 293, 299

Wilder, Billy 279

Wittgenstein, Ludwig 174

Witzigmann, Max 225

Wiwaldi 271ff., 277, 271ff., 277, 302, $324 f$.

Wollschläger, Hans 69,72

Wycherley, William 309

Zehrer, Klaus Cäsar 5, 8f., 67ff. 


\section{Sachindex}

Abweichung 22, 31, 37, 98, 102, 208, 216, 219, 294

- semantische Abweichung 34, 303

Agon 55

Akronym 52

Akrostichon 70

Alliteration 9, 37, 160, 226, 304

Alltagskommunikation 1f., 6, 106, 141, 187, 198, 201, 215, 225, 257, 265, 268, 278, 285, 287, 291, 296, 313

Alltagssprache Siehe Alltagskommunikation Ambiguität 3, 9f., 12f., 22, 49, 52, 56, 60, 65, $69,71,93,108,116,118,126,137,161$, $163,168,177 f ., 181,185,197,213,224$, 227, 232, 236, 254, 262, 270, 274, 279f., 285, 287, 295f., 298, 300ff., 309, 311f., 319

Anagramm 9, 59, 72, 168, 241

Angemessenheit 1, 10,132, 141, 171, 286f., 310

Anspielung 133, 173, 202

Aphorismus 12, 143f., 146, 149f., 153f., 203, 292

Arbitrarität 288ff., 292

Artikulation 12, 96ff., 101f., 215

Assonanz 52, 107

Assoziation 31, 49, 149, 180, 199, 224ff., $277,306 \mathrm{f}$.

Battle 55, 280

Bauchreden 12, $95 \mathrm{ff}$.

Bibel 117, 143f., 150, 153, 311

Bilderrätsel 272, 281, 302f., 307

Bildwitz 274

Binnenreim 135, 196, 199

Brief 13, 146, 149f., 187, $255 \mathrm{ff}$.

\section{Cartoon 2, 274}

Comedy 2, 5, 49, 105, 139, 157, 160, 223, 231, $242 \mathrm{f}$.

connivence $123,308 \mathrm{f}$.

Dadaismus, Dada 11, 55, 57, 60f., 64f., 187, 237
Deidiomatisierung 42, 128

Deutungsspielräume 13, 126, 285

Dialekt 71, 102, 132, 136, 146, 159, 174, 187, 225

Doppelbedeutung Siehe Ambiguität

Doppeldeutigkeit Siehe Ambiguität

Doppelsinnigkeit Siehe Ambiguität

Doubletime 55

Durchsichtigkeit 144ff., 152, 285

Etymologie 90, 143, 147, 152, 290f., 314, 319

Expressionismus 48, 55, 58

Figurengedicht Siehe Gedicht

Frame 127

Fremdsprache 3f., 7f., 25, 52f., 62, 71f., 102, 111, 118f., 134, 159, 161, 165, 171, 189ff., 201, 227, 236, 244, 250, 275, 280, 301f.

garden-path sentence Siehe Gartenpfadsatz Gartenpfadsatz 178, 306, 317

Gedicht 12, 19, 21f., 31f., 34ff., 40, 42, 45, 50, 56f., 59, 61, 64f., 67f., 70, 106f., 140f., 143f., 146, 148, 152, 159f., 165, 167, 172ff., 178f., 181f., 185, 187, 190f., 193, 195ff., 202f., 226, 234, 244ff., 253, 281, 307f., 313

- Figurengedicht 302

- Lautgedicht 64

Gleichklang Siehe Homophonie

Homoiophonie, Homoiophon 208, 213f., 253 Homonymie, Homonym 28, 144, 159, 191 Homophonie, Homophon 130, 159, 168, 181, 207, 213, 217, 250, 253, 299, 317

Humor $76,78,111,121,123,139,158,170 f$. , 224ff., 229, 241, 305

Idiom, idiomatisch 12, 123f., 126, 133, 181, 285ff., 293, 296, 298, 316 Improvisation 5, 231, 243, 271, 277 Inspiration 20, 48f., 53, 70, 77, 107, 117, 139 , 141, 159, 167, 169, 173ff., 187, 198, 225, 234, 244, 274 
Intertextualität 42, 91, 133

inventio 82

Ironie 2, 90, 121, 125f., 130, 136, 181, 218, $235,242,260,308$

Irritation 82, 119, 170, 253, 287

Jargon 47

Kabarett 2, 5, 71, 75, 83, 86, 90, 95, 102, 105f., 113ff., 139f., 157, 161, 167, 185f., 189, 192, 205, 215, 231, 243, 273

Kalauer 2, 74, 163, 173, 229, 241, 253, $279 f$. kataphorisch 206, 216

Kinderbuch, Kinderliteratur 2, 5, 185f., 188, 195ff., 203

Klang 1, 8, 21, 26, 47ff., 59, 72, 76, 118, 158f., $161,165,168,187,232,234,253,304$, 318

Klangfigur 4

Klangspiel 304

Knittelvers 12, 246, 253, 313

Komik 12, 67f., 70, 72ff., 106f., 110f., 121ff., 130ff., 136, 143, 157ff., 177ff., 198, 224ff., 237, 241ff., 249f., 265, 280, 300, 302, 313

- Situationskomik 242

Kommunikation 3, 6, 10, 13, 24, 51, 78, 105, 110ff., 118, 123, 141, 161, 170, 183, 190, 199, 235ff., 248f., 255, 260, 279, 286f., 292, 303, 309, 315

Kompositum 35ff., 41f., 52, 111, 121, 146, 148, 150, 181, 215, 248, 290, 292, 296ff., 306, 315

Kontext 1f., 6, 10ff., 28, 33ff., 40, 42, 52, 55, 72f., 80, 114, 118f., 123ff., 135f., 161, 163, 171, 173, 178f., 192, 203, 206, 216, 228f., 238, 251, 261, 264ff., 282, 287, 310, 318

Konzeptbereich 127

Kratylos 288, 292

Kreativität 3, 8, 26f., 31f., 39f., 54, 72, 79, 106, 112, 119, 140, 162, 171, 177f., 191, 195f., 198, 201, 203, 224, 228, 237, 281, 291

Lautgedicht Siehe Gedicht

Lautmalerei Siehe Onomatopoesie, Lautmalerei
Layout 61, 63, 65

Lettrismus 65

Lexikalisierung 36f., 39, 41f., 124, 127, 146, 254

Lied 9, 12, 48, 50, 105ff., 115f., 118, 123, 125, 128ff., 135, 139, 142, 160, 167, 225, 246. 285, 299, 309

Limerick 226, 238

Literalisierung 254

Macht 13, 29, 87, 190, 255, 261f., 265, 267

Manipulation 1, 12f., 51f., 54, 96, 98, 131f., 248, 265

McGurk-Effekt 101

Mehrdeutigkeit Siehe Ambiguität

Mehrfachreim 9,55

mehrsprachiges Wortspiel Siehe Wortspiel

Mehrsprachigkeit 7f., 25f., 52, 71, 79, 111, $118,161,171,190,201,227,233,236$. 244, 255, 268

Meistersinger 55, 149

Metapher 11, 31ff., 38ff., 56, 111, 149, 254f., 309, 313

Metaphernkomplex 40

metasprachliche Reflexion 180

Metrum, Metrik 54, 173, 181, 233

mise en abyme 129

Missverständnis 19, 25, 83, 111, 228, 286

Model Reader 256, 267

Motivation, Motivierung 13, 126, 145, 285 , 287ff., 295f., 304, 311f., 315, 319

- Motivierbarkeit 13, 129, 145, 248, 291, $310 f$.

- Pseudomotivation, Pseudomotivierung 9, 293f., 298f., 314

- Remotivation, Remotivierung 126, 293, $302 f$.

- Transmotivation, Transmotivierung 293

Mundart Siehe Dialekt

Name, Eigenname 12, 25, 121, 205ff., 213f., 216f., 219, 224, 233, 251, 275, 279, 288, 299, 315, 317

- Markenname 46

Narration $178,180 \mathrm{ff}$.

Narrativ 82, 93, 180, 182

Neologismus 48, 54, 143, 149, 151 
Onomatopoesie, Lautmalerei 64, 190, 201, 289, 304

Opakisierung 146f., 151

Palindrom 28, 72

Parodie 57, 83, 142, 160, 186f., 189f., 192

Paronymie 130, 180, 248, 250

Pathos 234

Pause Siehe Sprechpause

Performance 45, 112, 163, 231, 234, 292, 297

Personifikation 85, 178ff., 182

Phonetik 11f., 64, 103, 107, 112, 159, 180, 205, 207, 213f., 217f., 226f., 253, 307

Phraseologismus 122ff., 177ff.

Poetry Slam 5, 9, 55, 167, $178 f$.

Pointe $68,73,91,106 \mathrm{ff} ., 110,114,119 \mathrm{f} ., 128$, 135f., 139, 170, 191, 208f., 214ff., 224, 226, 242, 244, 248, 250, 274, 277f., 282, 285, 299, 305

Polyphonie 125ff., 131

Polysemie 144, 148, 150, 254, 261, 263, 265

Publikum 3, 5, 8, 15, 22, 48ff., 68, 71, 73, 75, $77,84,91,101 f ., 106,108,110,117,120$, 130, 134, 142, 158f., 161, 163, 169, 173, 188, 192, 198, 206, 209, 213ff., 218, 225, 227f., 234, 236, 266, 272

- Publikumsreaktion 13, 110, 127, 132, 136, 173, 205f., 209, 249, 307, 317

pun 227,253

Rap 9, 55, 171, 246

Reim 67ff., 72f., 107, 112, 116ff., 129ff., 139, 141, 143, 149, 157ff., 171, 174f., 181f., 187ff., 196, 199, 201, 233, 235f., 241f., 245f., 313

Reimschema 173, 182, 244

Reklame Siehe Werbung

Rekontextualisierung 205, $207 \mathrm{f}$.

Relexikalisierung 209, 217

Rhetorik 11, 66, 87, 150, 257

rhetorische Figur 55, 181

Rhythmus 22, 67ff., 234, 244

Satire 2, 71, 141, 192, 242

Schüttelreim 46, 52, 72, 117, 130, 168, 196, 226, 238, 241

Semiotik, semiotisch $61,64,66,137,256$
Situationskomik Siehe Komik

Sketch 46, 205f., 208, 210, 213, 216f., 225, 244ff., 274f., 277

Slam Poetry 49, 167

Sonett 9, 12, 246

Song Siehe Lied

Spitten 55

Spracharbeit 6f., 22, 49, 77, 107f., 117, 139, 141, 159, 169, 188, 198, 225, 234, 274

Sprachartistik Siehe Sprachkunst

Sprachexperiment 20

Sprachjonglage $49,68,76,157,165,224$, 253

Sprachkritik 314

Sprachkunst 4ff., 11f., 15, 22, 68, 139ff., 249 , 294

Sprachmagie 314

Sprachspiel 1ff., 10ff., 15, 21, 31, 48, 53, 57, 67ff., 81, 85, 88, 91f., 102, 122f., 131, $136,141,154,186,189,200,249,287$, 292, 294ff., 301ff., 308, 310, 315

Sprecher-Hörer-Interaktion 2f., 6, 13f., 137, 249ff., 303, 307, 315

Sprechpause 110, 132, 136, 179, 215f., 219, 249, 317

Strategie 13, 97f., 101, 139, 206, 261f., 265f., 268

- Interpretationsstrategie 11

- Sprecherstrategie 3

Subversion, subversiv 287, 319

Tabu 7, 111, 127f., 130, 134, 136, 286

Textualität 11, 57, 62, 65, 172

Tradition 28, 54f., 71, 73, 79, 81f., 95, 112ff., $119,121,141,145,162,172,179,192$, 201, 228, 238, 260, 264f., $302 f$.

Twitter 1, 54

- Live-Tweet 5

Typographie 63, 65, 82, 219

Übersetzung 3, 7f., 21, 23, 25, 32, 52, 69, 71f., 79, 111, 118, 149, 153, 161, 171, 190f., 201, 227, 236f., 244, 280, 283, 289f., 295, 302f., 306f., 309

Unverträglichkeit, Unvereinbarkeit 33, 35, 130,314 
Variation 12, 97, 122f., 129f., 134, 136, 171, 247f., 285, 299, 318

Verfremdung 1, 8, 122, 148, 185, 205, 301

Versmaß 107, 181, 244

Vieldeutigkeit Siehe Ambiguität

Wahrheit 56, 58, 83, 313f.

Werbung 1, 48, 56, 73, 171, 228, 238, 302

Wiederholung 12, 47, 98, 102, 110, 121ff., $125,127,129 \mathrm{ff} ., 134,136,152,178$, 247ff., 263ff., 285, 299

Witz 1f., 46, 69, 71f., 76, 121f., 142, 159, 161ff., 170, 203, 208, 227ff., 233, 241f., 249, 279ff., 293, 295, 299, 305

Wörterbuch 5, 7, 20f., 27, 77, 85f., 187, 250, 253, 306, 316

Wortfeld 12, 129, 181, 214

Wortkunst 55, 234, 236

Wortschatz 9, 12, 27, 54, 71, 86, 88, 112, 117, 119, 124, 161f., 171, 191, 201, 228, 236f., $245,248,282,289,315$
Wortschöpfung 52, 81, 153, 162, 168, 237, 245

Wortspiel

- geheimes Wortspiel 267, 308

- horizontales Wortspiel, Wortspiel in praesentia 13, 135, 178, 247, 249f., 307

- mehrsprachiges Wortspiel 3

- schlechtes Wortspiel 2, 10, 29, 46, 56, 74, $80,114,120,163,174,192,203,229$, 239

- vertikales Wortspiel, Wortspiel in absentia 13, 133, 178, 247ff., 307

\section{Zeugma 307}

Zufall 26, 49, 116, 186, 233, 241, 244, 253 , 264, 291f., 318

Zweideutigkeit Siehe Ambiguität

Zweisprachigkeit Siehe Mehrsprachigkeit 